\title{
Lipidomic analysis of fatty acids in erythrocytes of coeliac patients before and after a gluten-free diet intervention: a comparison with healthy subjects
}

\author{
Giuseppe Riezzo ${ }^{1}$, Carla Ferreri ${ }^{2}$, Antonella Orlando ${ }^{1}$, Manuela Martulli ${ }^{1}$, Benedetta D'Attoma ${ }^{1}$ and \\ Francesco Russo $^{1 *}$ \\ ${ }^{1}$ Laboratory of Nutritional Pathophysiology, National Institute for Digestive Diseases IRCCS 'Saverio de Bellis', \\ Via Turi 27, Castellana Grotte I-70013, Bari, Italy \\ ${ }^{2}$ Consiglio Nazionale delle Ricerche (CNR), ISOF Bio Free Radicals, Bologna, Italy
}

(Submitted 9 April 2014 - Final revision received 6 August 2014 - Accepted 7 August 2014 - First published online 30 September 2014)

\section{Abstract}

Coeliac disease (CD) patients may exhibit a pro-inflammatory profile and fatty acids (FA) can influence inflammation through a variety of cellular pathways in them. The aims of the present study were to (1) evaluate the FA composition of erythrocytes obtained from newly diagnosed CD patients by lipidomic analysis and compare it with that in healthy subjects and (2) determine the effects of 1-year gluten-free diet (GFD) intervention. A total of twenty CD patients (five men and fifteen women; mean age 34.0 (SEM 1.7) years) were evaluated at diagnosis and after 1 year of GFD intervention. A total of twenty healthy subjects (seven men and thirteen women; mean age $40 \cdot 2$ (SEM 2.5) years) served as controls. CD patients on an unrestricted diet exhibited a significant $2 \cdot 08$-fold higher concentration of arachidic acid when compared with healthy subjects, suggesting that it can be considered as a putative marker of CD. Besides, the arachidonic acid (AA): dihomo- $\gamma$-linolenic acid ratio was $2 \cdot 01$-fold significantly lower in CD patients than in healthy subjects $(P<0 \cdot 01)$, underlying an inefficient synthesis of PUFA from their precursors in terms of desaturase activity. In addition, mainly due to lower concentrations of docosahexaenoic acid, the inflammation marker AA:docosahexaenoic acid ratio was 1.40 -fold significantly higher in CD patients than in healthy subjects. After 1 year of GFD intervention, FA concentrations in CD patients were still different from those observed in healthy subjects. The lipidomic analysis of erythrocyte membranes confirmed the presence of an altered FA composition in CD patients and the GFD's ability to modify FA profile, even if 1-year GFD intervention seems to be not sufficient to restore FA concentrations to normality. This procedure, being easier and non-invasive compared with the evaluation of the FA pattern of the intestinal mucosa, could offer more potentiality for also evaluating therapeutic interventions in CD patients by using FA supplementation.

\section{Key words: Coeliac disease: Erythrocyte membranes: Fatty acids: Lipidomic analysis}

Coeliac disease (CD) is an autoimmune disorder characterised by villous atrophy of the upper small intestine caused by intolerance to gluten in wheat and similar proteins in barley and rye $^{(1)}$. CD can present with a wide spectrum of symptoms including both gastrointestinal (GI; e.g. diarrhoea, bloating and abdominal pain) and extra-GI symptoms. CD therapy involves a gluten-free diet (GFD), which allows CD patients to achieve functional and structural restoration of the intestinal mucosa ${ }^{(2)}$.

Different autoimmune diseases sharing a persistent low-grade inflammation (such as diabetes, Hashimoto's thyroiditis and psoriasis) exhibit an earlier atherosclerosis progression ${ }^{(3)}$. Consistent with the immune and inflammatory conditions of $\mathrm{CD}$, an inflammatory profile and an increased risk for the development of inflammation-related conditions might also be expected in CD patients ${ }^{(4-6)}$. In this context, there is growing evidence suggesting that excessive fatty acids (FA), namely
SFA, MUFA and PUFA and endogenous trans-FA, could play an active role ${ }^{(7)}$. Notably, such an increase in FA may be related to diet and radical stress conditions ${ }^{(8)}$.

Various dietary components including long-chain $n$-3 FA, plant flavonoids and carotenoids have been demonstrated to modulate oxidative stress, gene expression and inflammatory mediator production ${ }^{(9)}$. Besides, FA composition of intestinal membranes is important for epithelial function, and its disorders may contribute to the pathophysiology of CD.

The composition of dietary FA is reflected in circulating FA profile. Therefore, determination of FA in different biological samples could be used to diagnose deficiency of some FA, especially the essential FA (linoleic acid and $\alpha$-linolenic acid), in subjects adhering to restricted diets because of food allergies or other diseases. However, the constituents of complex lipids are extremely different, and the combinations

Abbreviations: AA, arachidonic acid; CD, coeliac disease; DGLA, dihomo- $\gamma$-linolenic acid; FA, fatty acids; GFD, gluten-free diet; GI, gastrointestinal.

*Corresponding author: Dr F. Russo, fax +39 0804994313, email francesco.russo@irccsdebellis.it 
and permutations at which they are assembled in individual molecular lipid species further strengthen this difference. Currently, an extensive list of lipid classes and their distinctive molecular species that are present in a biological sample can be obtained by lipidomic analysis and FA-based functional lipidomics is gaining an important place among the molecular diagnostic tools available to physicians ${ }^{(10,11)}$. Methodologically, the complexity of the task is exemplified by the human erythrocyte, which contains only a plasma membrane ${ }^{(12)}$. This membrane is known to contain up to 300 molecular species of glycerophospholipids formed by different long-chain (C14-C22) FA, each present at a level exceeding $2 \%$ of the total FA.

The content of FA in erythrocytes represents the result of interactions among dietary FA and endocrine and immunological modifications ${ }^{(13)}$, partly due to the long half-life $(120 \mathrm{~d})$ of erythrocytes. Under dietary restrictions that can reduce the inflammatory status and immune response ${ }^{(12)}$, an active role may be plausible for the individual FA pool. In this perspective, its evaluation could be useful for evaluating the relations with dietary restrictions due to a GFD and investigating the non-immunological mechanism of the gluten intolerance.

Contrasting data on FA profile in CD patients are available in the literature, and these have mainly been obtained from serum or tissue evaluations ${ }^{(4,14)}$, but not from lipidomic analysis of erythrocyte membranes. Thus, by applying this methodology, the aims of the present study were (1) to evaluate the FA composition of erythrocyte membranes in newly diagnosed CD patients and compare it with that in healthy subjects and (2) to investigate whether 1-year GFD intervention can induce changes in FA composition in CD patients.

\section{Methods}

\section{Participants}

Cases for the present case-control study were recruited from among the outpatients of the National Institute of Digestive Diseases, IRCCS 'Saverio de Bellis', Castellana G. (Italy).

They were screened and recruited according to the following inclusion criteria: age $>18$ years; active symptoms for at least 2 weeks; a minimum average of 3.0 on the seven-point Likert scale of the Gastrointestinal Symptom Rating Scale-irritable bowel syndrome composite symptom score; the assumption of a free diet.

Exclusion criteria included metabolic and endocrine disorders; hepatic diseases, renal diseases, or CVD; constipation; fever and intense physical activity; previous abdominal surgery; history of malignancy; secondary causes of intestinal atrophy; pregnancy and lactose intolerance; and giardiasis. In addition, cases were excluded if they reported the use of medication for the treatment of irritable bowel syndrome for 2 weeks before evaluation, antibiotics or probiotic agents, and other drugs known to cause abdominal pain. All the women were examined during the follicular phase of the menstrual cycle.

Cases were first diagnosed to have $\mathrm{CD}$ on the basis of both positive antitransglutaminase antibodies and antiendomysium antibodies. In the case of a positive serological test, biopsies from the proximal part and from the distal part of the duodenum were taken to confirm the diagnosis. Cases were considered to have $\mathrm{CD}$ if confirmed by histological evidence, according to the modified Marsh-Oberhuber criteria (grades 3b-3c) ${ }^{(15)}$.

After diagnosis, CD patients were instructed to follow a specific GFD as reported in Table 1 and given additional counselling during the follow-up visit. CD patients were evaluated at diagnosis and at least 12 months after the start of GFD intervention. Dietary compliance assessment during the 1-year visit was based on the assessment of coeliac serology, self-reported compliance, and the opinion of the patient's

Table 1. Dietary suggestions for standardised consumption of gluten-free products

\begin{tabular}{|c|c|c|c|}
\hline & Foods & Quantity/d & Nutritional facts $(100 \mathrm{~g})$ \\
\hline \multirow[t]{2}{*}{ Breakfast } & $\begin{array}{l}\text { Twice-baked } \\
\text { sliced bread } \\
\text { (or) }\end{array}$ & $\begin{array}{l}\text { Four } \\
\quad \text { slices }(32 \mathrm{~g})\end{array}$ & $\begin{array}{l}\text { Energy } 1719 \mathrm{~kJ} \\
\text { Protein } 2 \cdot 2 \mathrm{~g} \\
\text { Fat } 6 \cdot 2 \mathrm{~g} \\
\text { Saturated fat } 3.4 \mathrm{~g} \\
\text { Carbohydrates } 83.0 \mathrm{~g} \\
\text { Sugar } 5.2 \mathrm{~g} \\
\text { Fibre } 5.2 \mathrm{~g}\end{array}$ \\
\hline & Biscuits & $\begin{array}{l}\text { Four } \\
\quad \text { biscuits }(28 \mathrm{~g})\end{array}$ & $\begin{array}{l}\text { Energy } 1896 \mathrm{~kJ} \\
\text { Protein } 5.4 \mathrm{~g} \\
\text { Fat } 14.7 \mathrm{~g} \\
\text { Saturated fat } 7.8 \\
\text { Carbohydrates } 72.0 \mathrm{~g} \\
\text { Sugar } 21.0 \mathrm{~g} \\
\text { Fibre } 3.1 \mathrm{~g}\end{array}$ \\
\hline \multirow[t]{2}{*}{ Lunch } & Pasta* $^{*}$ & A portion $(80 \mathrm{~g})$ & $\begin{array}{l}\text { Energy } 1539 \mathrm{~kJ} \\
\text { Protein } 8.3 \mathrm{~g} \\
\text { Fat } 1.5 \mathrm{~g} \\
\text { Saturated fat } 0.4 \mathrm{~g} \\
\text { Carbohydrates } 78.0 \mathrm{~g} \\
\text { Sugar } 4.4 \mathrm{~g} \\
\text { Fibre } 2.0 \mathrm{~g}\end{array}$ \\
\hline & $\begin{array}{l}\text { Homemade } \\
\text { bread }\end{array}$ & One slice (48g) & $\begin{array}{l}\text { Energy } 966 \mathrm{~kJ} \\
\text { Protein } 3.4 \mathrm{~g} \\
\text { Fat } 3.5 \mathrm{~g} \\
\text { Saturated fat } 0.5 \mathrm{~g} \\
\text { Carbohydrates } 43.0 \mathrm{~g} \\
\text { Sugar } 3.0 \mathrm{~g} \\
\text { Fibre } 5.7 \mathrm{~g}\end{array}$ \\
\hline Snack & Crackers & A portion $(35 \mathrm{~g})$ & $\begin{array}{l}\text { Energy } 1844 \mathrm{~kJ} \\
\text { Protein } 4.5 \mathrm{~g} \\
\text { Fat } 10.8 \mathrm{~g} \\
\text { Saturated fat } 6.2 \mathrm{~g} \\
\text { Carbohydrates } 78.6 \mathrm{~g} \\
\text { Sugar } 3.5 \mathrm{~g} \\
\text { Fibre } 2.2 \mathrm{~g}\end{array}$ \\
\hline \multirow[t]{2}{*}{ Dinner } & Pasta* $^{*}$ & A portion $(40 \mathrm{~g})$ & $\begin{array}{l}\text { Energy } 770 \mathrm{~kJ} \\
\text { Protein } 4.1 \mathrm{~g} \\
\text { Fat } 0.7 \mathrm{~g} \\
\text { Saturated fat } 0.2 \mathrm{~g} \\
\text { Carbohydrates } 39.0 \mathrm{~g} \\
\text { Sugar } 2.2 \mathrm{~g} \\
\text { Fibre } 1.0 \mathrm{~g}\end{array}$ \\
\hline & Sliced bread & $\begin{array}{l}\text { Three } \\
\quad \text { slices }(60 \mathrm{~g})\end{array}$ & $\begin{array}{l}\text { Energy } 965 \mathrm{~kJ} \\
\text { Protein } 2.9 \mathrm{~g} \\
\text { Fat } 5.0 \mathrm{~g} \\
\text { Saturated fat } 0.7 \mathrm{~g} \\
\text { Carbohydrates } 39.7 \mathrm{~g} \\
\text { Sugar } 3.8 \mathrm{~g} \\
\text { Fibre } 6.3 \mathrm{~g}\end{array}$ \\
\hline
\end{tabular}

${ }^{*}$ Pasta could be substituted by naturally gluten-free food. 
physician. The physicians categorised each patient's compliance with the GFD as strict compliance; partial compliance with occasional lapses (i.e. two to three lapses per month or no more than one lapse per week); and non-compliance or no gluten restriction at all. When discrepancy was observed among the assessments, the physician reassessed the patient to determine the final categorisation.

As controls, healthy subjects were enrolled from among the administrative staff of our institute. They reported that they did not have metabolic, endocrine or immunological diseases, dyspepsia, or other GI diseases and did not take any medication. Information on the health status of these subjects was obtained during an examination consisting of an interview on the current diet, lifestyle, and medical history and a physical examination. Negative antiendomysium antibodies and antitransglutaminase antibodies were criteria for inclusion in the study. Besides, metabolic parameters (blood glucose, glycated $\mathrm{Hb}$, lipid profile, body weight and blood pressure) had to be in the normal range.

The present study was conducted according to the guidelines laid down in the Declaration of Helsinki, and all procedures involving human subjects/patients were approved by the local Scientific and Ethics Committees. Written informed consent was obtained from all the patients and healthy subjects. The clinical trial on CD was registered at http://www.clinicaltrials. gov (register no. NCT01574209).

\section{Analytical measurements}

All the analytical measurements were performed at the time of enrolment. In CD patients, the measurements were repeated during the follow-up visit, after being on the GFD intervention for at least 12 months.

A blood sample was collected after an overnight fast, immediately centrifuged and stored at $-80^{\circ} \mathrm{C}$ until analysis. Routine biochemical analyses were conducted to evaluate the values of fasting serum total cholesterol (mmol/l), HDL (mmol/l), LDL:HDL ratio, TAG (mmol/l), ferritin ( $\mathrm{mg} / \mathrm{l})$, erythrocyte sedimentation rate $(\mathrm{mm} / \mathrm{h})$, C-reactive protein $(\mathrm{mg} / \mathrm{l})$, leucocyte count $\left(10^{9} / 1\right)$ and platelet count $\left(10^{9} / 1\right)$. LDL values were calculated according to the Friedewald formula ${ }^{(16)}$.

Antiendomysium antibodies were detected using an indirect immunofluorescence technique and antitransglutaminase antibodies using ELISA (Eurospital).

The plasma concentrations of IL- 6 and IL- 8 were measured in duplicate using commercially available sandwich ELISA kits (Human IL-6 ELISA and Human IL-8 ELISA; BD Biosciences).

\section{Erythrocyte isolation}

In both CD patients and controls, a blood sample was collected from the cubital vein into an evacuated blood collection tube containing EDTA. Erythrocytes were separated using Ficoll-Hypaque (Pharmacia) density gradient centrifugation. The pellet was washed three times with four volumes of $0.9 \% \mathrm{NaCl}$ and then erythrocytes in the pellet aliquot were counted using a Sysmex-Toak 1000 cell counter (Gelman Instrument). Each sample was stored at $-80^{\circ} \mathrm{C}$ until analysis.

\section{Lipidomic analysis}

Lipidomic analysis was performed by Lipinutragen srl (Bologna, Italy). The total lipid fractions, obtained by fractionation of erythrocytes from the study groups, were extracted with 2:1 chloroform-methanol $(\mathrm{v} / \mathrm{v})^{(17)}$, and the extracts were examined by TLC for the presence of lipid classes, as described previously, which were identified by reference compounds to be phospholipids mixed with $\mathrm{TAG}^{(18)}$. The extracts were treated by transesterification with $\mathrm{KOH} / \mathrm{MeOH}(0.5 \mathrm{M})$ for $20 \mathrm{~min}$ at room temperature ${ }^{(19)}$. FA methyl esters were subsequently extracted with $n$-hexane and $1 \mu \mathrm{l}$ of these solutions was used for analysis.

FA methyl esters were analysed by GC (Agilent 6850) equipped with a $60 \mathrm{~m} \times 0.25 \mathrm{~mm} \times 0.25 \mu \mathrm{m}$ (50\%-cyanopropyl)methylpolysiloxane column (DB23, Agilent) and a flame ionisation detector. Injector temperature was $230^{\circ} \mathrm{C}$.

The oven conditions were as follows: temperature started from $195^{\circ} \mathrm{C}$, held for $26 \mathrm{~min}$, followed by an increase of $10^{\circ} \mathrm{C} /$ min up to $205^{\circ} \mathrm{C}$, held for $13 \mathrm{~min}$, followed by a second increase of $30^{\circ} \mathrm{C} / \mathrm{min}$ up to $240^{\circ} \mathrm{C}$, and held for $10 \mathrm{~min}$. A constant pressure mode ( 29 pounds per square inch) was chosen with hydrogen as the carrier gas.

Methyl esters were identified by comparison with the retention times of authentic samples. GC/MS spectra were recorded on a Clarus 500 GC apparatus equipped with a Clarus $560 \mathrm{~S}$ mass spectrometer (GC/MS transfer line temperature $230^{\circ} \mathrm{C}$ (Perkin Elmer)) and a $60 \mathrm{~m} \times 0.25 \mathrm{~mm} \times 0.25 \mu \mathrm{m}(50 \%-$ cyanopropylphenyl)-dimethylpolysiloxane column (DB225ms, Agilent), using an injector temperature of $230^{\circ} \mathrm{C}$, a split ratio of 50:1 and helium as the carrier gas at a constant flow of $1.2 \mathrm{ml} / \mathrm{min}$, with the following oven conditions: temperature started from $195^{\circ} \mathrm{C}$, held for $52 \mathrm{~min}$, followed by an increase of $3^{\circ} \mathrm{C} / \mathrm{min}$ up to $205^{\circ} \mathrm{C}$, held for $10 \mathrm{~min}$, followed by a second increase of $3^{\circ} \mathrm{C} / \mathrm{min}$ up to $225^{\circ} \mathrm{C}$, held for $15 \mathrm{~min}$, and a final increase of $5^{\circ} \mathrm{C} / \mathrm{min}$ up to $230^{\circ} \mathrm{C}$ and held for $10 \mathrm{~min}$.

\section{Sample size calculation}

The present study aimed to detect a change in the FA profile considering that at least $75 \%$ of the patients would report the absence of diarrhoea after 1 year of GFD intervention as detected by the Gastrointestinal Symptom Rating Scale cluster 'diarrhoea syndrome' (three items: increased frequency of evacuation, loose stools and urgent need to defaecate; maximum score: 21).

Considering a magnitude of the difference between the means in cluster 'diarrhoea syndrome' of 3.7 and a standard deviation of 4.0 at a significance level of 0.05 (two tailed), nineteen patients per group were required to have $80 \%$ power to detect a significant difference between the study groups.

\section{Statistical analysis}

All results are expressed as means with their standard errors unless otherwise specified. The Kruskal-Wallis test (with Dunn's post hoc test) was used to assess differences among the study groups. 


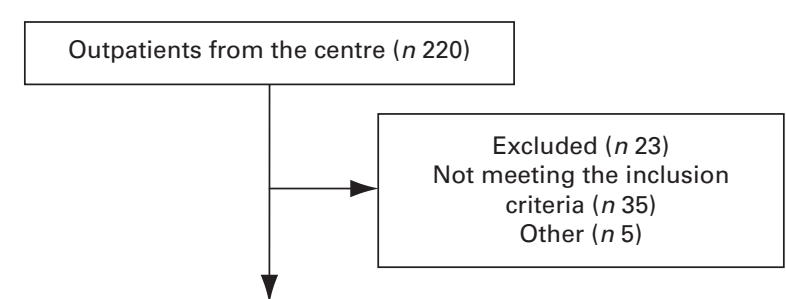

Patients fulfilling the inclusion criteria (n 157)
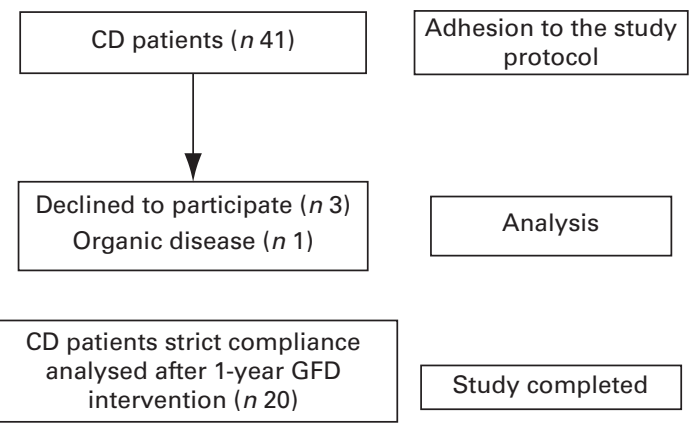

Study completed

Fig. 1. The flow of participants through the study. CD, coeliac disease; GFD, gluten-free diet.

All the differences were considered significant at the 5\% level. A specific statistical package for exact non-parametric inference (Stata Statistical Software: Release 9; StataCorp, 2005) was used.

\section{Results}

\section{Participant characteristics}

The flow of participants through the study is shown in Fig. 1. A total 220 individuals with GI symptoms resembling irritable bowel syndrome were recruited between January 2010 and November 2012. Of these patients, thirty-five did not fulfil the inclusion criteria; twenty-three were excluded due to

refusal to undergo endoscopy or refusal to adhere to dietary requirements; and five did not participate in the study due to other reasons. A total of 157 patients met the inclusion criteria, and forty-one adult newly diagnosed CD patients with diarrhoea as the prevalent GI symptom were identified. Among these patients, three declined to participate and one had an organic disease. After 1 year of GFD intervention, twenty CD patients were found to have strictly complied to the dietary requirements (five men and fifteen women; mean age 34.0 (SEM 1.7) years; mean BMI 22.5 (SEM 0.5) kg/m²) and were included in the analysis. These patients were evaluated at diagnosis and at least 12 months after the start of GFD intervention (13.6 (SEM 1.2) months). The healthy control group comprised twenty-eight subjects; among these, eight did not complete the study. Thus, twenty healthy subjects (seven men and thirteen women; mean age $40 \cdot 2$ (SEM 2.5) years; mean BMI $24.8($ SEm 0.8$) \mathrm{kg} / \mathrm{m}^{2}$ ) completed the study. The anthropometric and medical data are reported in Table 2.

Apart from diarrhoea, the main presenting GI symptoms in CD patients were abdominal pain (11.0\%) and dyspepsia (4.6\%). Villous atrophy was partial in $45 \%$ and total in $55 \%$. The most common non-GI symptoms in the majority of CD cases were anaemia (20.7\%) and osteopaenia (6.0\%). Follow-up blood samples were obtained during the 1-year follow-up visit.

Serum lipid concentrations at baseline and after the GFD intervention are reported in Table 3. Either before or after 1 year of GFD intervention, CD patients exhibited significantly lower total cholesterol $(P=0.004)$, LDL $(P=0.004)$ and TAG $(P=0.005)$ concentrations and LDL:HDL ratio $(P=0.006)$ in comparison with healthy subjects. There were no significant differences in HDL concentrations among the study groups. Besides, after 1 year of GFD intervention, all the lipidic variables in $C D$ patients remained substantially unchanged (data analysed by Kruskal-Wallis test with Dunn's multiple comparison test). There was a $4.4 \mathrm{~kg}$ increase in the mean weight (95\% CI $2 \cdot 7,5 \cdot 35$ ) from the baseline mean weight of $66 \cdot 9$ (SD $4 \cdot 0) \mathrm{kg}$.

Table 2. Anthropometric and medical data of healthy controls and coeliac disease (CD) patients at diagnosis and after 1 year of gluten-free diet (GFD) intervention*

(Mean values with their standard errors; $n 20$ )

\begin{tabular}{|c|c|c|c|c|}
\hline & Healthy controls & $\begin{array}{l}\text { CD patients } \\
\text { at diagnosis }\end{array}$ & $\begin{array}{l}\text { CD patients after } \\
\text { GFD intervention }\end{array}$ & $P$ \\
\hline Smokers & 4 & 5 & 4 & 0.90 \\
\hline Alcohol intake & & & & 0.92 \\
\hline No & 15 & 14 & 14 & \\
\hline $1-2$ glasses/d & 5 & 6 & 6 & \\
\hline$>2$ glasses $/ \mathrm{d}$ & 0 & 0 & 0 & \\
\hline BMI $\left(\mathrm{kg} / \mathrm{m}^{2}\right)$ & & & & 0.32 \\
\hline Mean & $24 \cdot 8$ & 22.5 & $23 \cdot 2$ & \\
\hline SEM & 0.8 & 0.5 & 1.0 & \\
\hline Systolic blood pressure $(\mathrm{mmHg})$ & & & & 0.53 \\
\hline Mean & $120 \cdot 5$ & $119 \cdot 3$ & $115 \cdot 9$ & \\
\hline SEM & $2 \cdot 0$ & $3 \cdot 0$ & $2 \cdot 9$ & \\
\hline Diastolic blood pressure $(\mathrm{mmHg})$ & & & & 0.38 \\
\hline Mean & $72 \cdot 7$ & $69 \cdot 7$ & $72 \cdot 2$ & \\
\hline SEM & 1.8 & 1.8 & 1.6 & \\
\hline Number of co-morbid diseases & 1 & 1 & 1 & 1.0 \\
\hline Physical activity (yes) & 8 & 9 & 8 & 0.93 \\
\hline
\end{tabular}

*Evaluated using the Kruskal-Wallis test and Dunn's multiple comparison test and the $\chi^{2}$ test for categorical variables. 
Table 3. Serum lipid concentrations in healthy controls and coeliac disease (CD) patients at diagnosis and after 1 year of gluten-free diet (GFD) intervention*

(Mean values with their standard errors; $n$ 20)

\begin{tabular}{|c|c|c|c|c|c|c|c|}
\hline & \multicolumn{2}{|c|}{$\begin{array}{l}\text { Healthy } \\
\text { controls }\end{array}$} & \multicolumn{2}{|c|}{$\begin{array}{l}C D \text { patients } \\
\text { at diagnosis }\end{array}$} & \multicolumn{2}{|c|}{$\begin{array}{l}\text { CD patients } \\
\text { after GFD } \\
\text { intervention }\end{array}$} & \multirow[b]{2}{*}{$P$} \\
\hline & Mean & SEM & Mean & SEM & Mean & SEM & \\
\hline Total cholesterol $(\mathrm{mmol} / \mathrm{l})$ & $4 \cdot 78^{\mathrm{a}}$ & 0.19 & $3 \cdot 75^{b}$ & 0.16 & $3 \cdot 66^{b}$ & 0.08 & $<0.0001$ \\
\hline $\mathrm{HDL}(\mathrm{mmol} / \mathrm{l})$ & $1 \cdot 28^{a}$ & 0.06 & $1 \cdot 14^{\mathrm{b}}$ & 0.03 & $1 \cdot 21^{\mathrm{a}}$ & 0.04 & 0.045 \\
\hline $\mathrm{LDL}(\mathrm{mmol} / \mathrm{l})$ & $2 \cdot 91^{\mathrm{a}}$ & 0.18 & $2 \cdot 18^{\mathrm{b}}$ & 0.15 & $2 \cdot 02^{b}$ & 0.08 & 0.003 \\
\hline LDL:HDL ratio & $2 \cdot 32^{\mathrm{a}}$ & 0.15 & $1.93^{b}$ & 0.14 & $1.69^{b}$ & 0.05 & 0.01 \\
\hline $\mathrm{TAG}(\mathrm{mmol} / \mathrm{l})$ & $1 \cdot 30^{\mathrm{a}}$ & 0.16 & $0.94^{\mathrm{b}}$ & 0.05 & $0.94^{\mathrm{b}}$ & 0.06 & 0.01 \\
\hline
\end{tabular}

${ }^{\mathrm{a}, \mathrm{b}}$ Mean values within a row with unlike superscript letters were significantly different $(P<0.05$; Dunn's test).

*Evaluated by the Kruskal-Wallis test and Dunn's multiple comparison test.

Inflammatory markers in coeliac disease patients before and after 1 year of g/uten-free diet intervention and healthy controls

The mean values of erythrocyte sedimentation rate and C-reactive protein concentrations were significantly higher in CD patients, either before or after 1 year of GFD intervention, than those in healthy subjects, but there were no significant differences in leucocyte and platelet counts and ferritin concentrations (Table 4).

The plasma concentrations of IL- 6 and IL-8 in healthy subjects and $\mathrm{CD}$ patients, either before or after 1 year of GFD intervention, are shown in Fig. 2 (a) and (b), respectively. The mean plasma IL-6 concentration was 3.78 (SEM $0.52) \mathrm{pg} / \mathrm{ml}$ in healthy subjects, 5.48 ( patients on an unrestricted diet and 2.89 (SEM 0.63$) \mathrm{pg} / \mathrm{ml}$ in CD patients on a GFD for 1 year. There were significant differences in IL- 6 concentrations among the study groups $(P=0.001)$. In response to the GFD intervention, IL- 6 concentrations in CD patients decreased by $48.7 \%$ when compared with the initial values, and Dunn's test showed that there were significant differences in the IL- 6 concentrations of $\mathrm{CD}$ patients before and after 1 year of GFD intervention $(P<0 \cdot 05)$.

The mean plasma IL-8 concentration was 9.49 (SEM $1.59) \mathrm{pg} / \mathrm{ml}$ in healthy subjects, 28.51 (SEM 7.73 ) $\mathrm{pg} / \mathrm{ml}$ in CD patients on an unrestricted diet and 10.87 (SEM 1.56$) \mathrm{pg} / \mathrm{ml}$ in $\mathrm{CD}$ patients on a GFD for 1 year. In addition, there were significant differences in IL-8 concentrations among the study groups $(P<0 \cdot 0001)$. After 1 year of dietetic treatment, IL-8 concentrations decreased in CD patients by almost $62 \%$ compared with the initial values, reaching that found in healthy subjects. Dunn's test showed that IL-8 concentrations in CD patients on an unrestricted diet were significantly different from those in both healthy subjects and $\mathrm{CD}$ patients on a GFD $(P<0.05$; data analysed by Kruskal-Wallis test with Dunn's multiple comparison test).

\section{Fatty acid profile}

The lipidomic profile of the erythrocyte membranes of $\mathrm{CD}$ patients is summarised in Table 5. When compared with that of healthy subjects, the lipidomic profile of $\mathrm{CD}$ patients exhibited significantly lower concentrations of palmitic acid (16: $0 ; P=0.02)$, stearic acid $(18: 0 ; P=0.008)$, oleic acid $(18: 1$; $P=0.04)$, linoleic acid $(18: 2 ; P=0.01)$ and DHA (22:6n-3; $P=0.04)$ and higher concentrations of arachidic acid (20:0; $P<0 \cdot 0001)$, eicosadienoic acid (20:2;P=0.03), dihomo- $\gamma$-linolenic acid (DGLA; $20: 3 n-6 ; P=0 \cdot 01$ ), eicosatrienoic acid (20: $3 n-3 ; P=0.04)$, docosapentaenoic acid $(22: 5 n-3 ; P=0.0005)$ and $(20: 4 t)$ FA $(P=0 \cdot 009)$.

As regards SFA, the concentrations of arachidic acid (20:0) were 2.08-fold higher in CD patients, suggesting that this SFA can be considered as a peculiar marker of active disease.

Table 4. Markers of systemic inflammation in healthy controls and coeliac disease (CD) patients at diagnosis and after 1 year of gluten-free diet (GFD) intervention*

(Mean values with their standard errors, $n$ 20)

\begin{tabular}{|c|c|c|c|c|c|c|c|}
\hline & \multicolumn{2}{|c|}{$\begin{array}{l}\text { Healthy } \\
\text { controls }\end{array}$} & \multicolumn{2}{|c|}{$\begin{array}{l}\text { CD patients } \\
\text { at diagnosis }\end{array}$} & \multicolumn{2}{|c|}{$\begin{array}{l}\text { CD patients after } \\
\text { GFD intervention }\end{array}$} & \multirow[b]{2}{*}{$P$} \\
\hline & Mean & SEM & Mean & SEM & Mean & SEM & \\
\hline $\operatorname{ESR}(\mathrm{mm} / \mathrm{h})$ & $10 \cdot 44^{\mathrm{a}}$ & $1 \cdot 14$ & $19 \cdot 06^{b}$ & 2.59 & $12 \cdot 04^{\mathrm{a}, \mathrm{b}}$ & 0.59 & 0.019 \\
\hline C-reactive protein (mg/l) & $1.77^{\mathrm{a}}$ & 0.26 & $4 \cdot 71^{\mathrm{b}}$ & 0.49 & $4.48^{\mathrm{b}}$ & 0.51 & $<0.0001$ \\
\hline Leucocyte count $\left(10^{9} / I\right)$ & $6060^{a}$ & $279 \cdot 3$ & $6340^{a}$ & $525 \cdot 0$ & $6187^{a}$ & $412 \cdot 0$ & 0.938 \\
\hline Platelet count $\left(10^{9} / \mathrm{l}\right)$ & $236800^{a}$ & 16206 & $268625^{a}$ & 22386 & $230375^{a}$ & 16251 & 0.299 \\
\hline Ferritin (mg/l) & $59 \cdot 79^{a}$ & 14.33 & $29 \cdot 14^{\mathrm{a}}$ & $7 \cdot 66$ & $37 \cdot 06^{\mathrm{a}}$ & 4.82 & 0.207 \\
\hline
\end{tabular}

ESR, erythrocyte sedimentation rate.

${ }^{a, b}$ Mean values within a row with unlike superscript letters were significantly different $(P<0.05$; Dunn's test).

*Evaluated by the Kruskal-Wallis test and Dunn's multiple comparison test. 
(a)

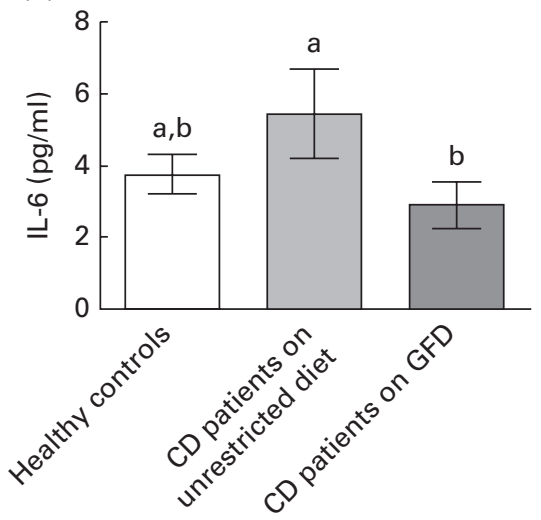

(b)

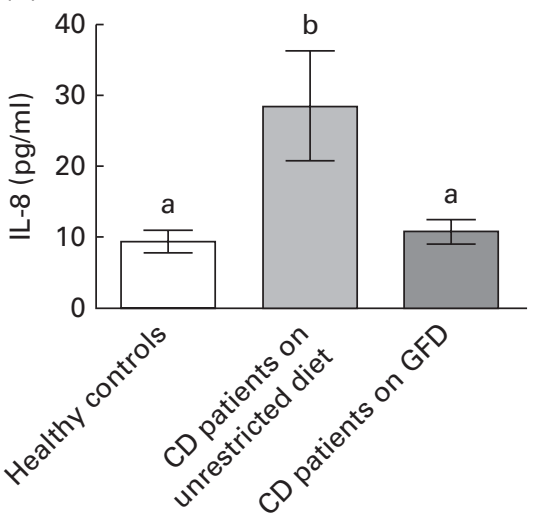

Fig. 2. Distribution of IL-6 (a) and IL-8 (b) in healthy subjects $(n$ 20) and coeliac disease (CD) patients on an unrestricted diet $(n 20)$ and after 1 year of gluten-free diet (GFD) intervention ( $n$ 20). Values are means, with their standard errors represented by vertical bars (Kruskal-Wallis test and Dunn's post hoc test). ${ }^{a, b}$ Mean values with unlike superscript letters were significantly different $(P<0.05$; Dunn's test).

SFA are substrates of the $\Delta$ 9-desaturase enzymes: stearoyl-CoA desaturase-1 and -2 . These rate-limiting enzymes transform the SFA palmitic and stearic acids into palmitoleic and oleic acids, respectively ${ }^{(20)}$. Their activity can be represented by the
$\Delta$ 9-desaturase index (oleic acid:stearic acid ratio); its reciprocal, defined saturation index, can be considered an indicator of membrane fluidity. In CD patients on an unrestricted diet, SFA were mainly converted into MUFA, as palmitic acid (16:0) was

Table 5. Lipidomic profile of erythrocyte membranes of healthy controls and coeliac disease (CD) patients at diagnosis and after 1 year of gluten-free diet (GFD) intervention*

(Mean values with their standard errors, $n 20$ )

\begin{tabular}{|c|c|c|c|c|c|c|c|c|c|}
\hline \multirow[b]{2}{*}{ Fatty acids } & \multirow[b]{2}{*}{ Lipid names } & \multicolumn{2}{|c|}{ Healthy controls } & \multicolumn{3}{|c|}{ CD at diagnosis } & \multicolumn{2}{|c|}{ CD on GFD } & \multirow[b]{2}{*}{$P$} \\
\hline & & Mean & SEM & Mean & SEM & Fold change $†$ & Mean & SEM & \\
\hline SFA & & $50 \cdot 74^{a}$ & 4.99 & $43 \cdot 82^{b}$ & $6 \cdot 35$ & $-1 \cdot 16$ & $46 \cdot 05^{\mathrm{a}, \mathrm{b}}$ & 6.48 & 0.003 \\
\hline Palmitic acid & $16: 0$ & $32 \cdot 71^{a}$ & $4 \cdot 08$ & $27 \cdot 43^{b}$ & 4.90 & $-1 \cdot 19$ & $28.94^{b}$ & 4.96 & 0.02 \\
\hline Heptadecanoic acid & $17: 0$ & $1 \cdot 84^{a}$ & 0.49 & $1.57^{\mathrm{a}}$ & 0.47 & $-1 \cdot 17$ & $1.67^{\mathrm{a}}$ & 0.68 & 0.45 \\
\hline Stearic acid & $18: 0$ & $15 \cdot 82^{\mathrm{a}}$ & $1 \cdot 32$ & $14 \cdot 05^{\mathrm{b}}$ & 1.55 & $-1 \cdot 13$ & $14 \cdot 89^{a, b}$ & $2 \cdot 23$ & 0.008 \\
\hline Arachidic acid & $20: 0$ & $0.37^{a}$ & 0.12 & $0.77^{\mathrm{b}}$ & 0.24 & +2.08 & $0.53^{a}$ & 0.23 & $<0.0001$ \\
\hline MUFA & & $19 \cdot 95^{a}$ & $3 \cdot 18$ & $18 \cdot 06^{b}$ & 3.09 & $-1 \cdot 10$ & $17 \cdot 95^{\mathrm{a}, \mathrm{b}}$ & 2.52 & 0.02 \\
\hline Palmitoleic acid & $16: 1 n-7 c$ & $1 \cdot 24^{\mathrm{a}}$ & 0.52 & $1.52^{\mathrm{a}}$ & 0.71 & $+1 \cdot 22$ & $1 \cdot 29^{a}$ & 0.53 & 0.15 \\
\hline Oleic acid & $18: 1 n-9 c$ & $16 \cdot 27^{a}$ & 2.07 & $14 \cdot 67^{b}$ & $2 \cdot 33$ & $-1 \cdot 11$ & $14 \cdot 99^{\mathrm{a}, \mathrm{b}}$ & $2 \cdot 32$ & 0.04 \\
\hline Cis-vaccenic acid & $18: 1 n-11 c$ & $1.47^{\mathrm{a}}$ & 0.20 & $1 \cdot 36^{a, b}$ & 0.30 & -1.08 & $1 \cdot 27^{\mathrm{b}}$ & 0.19 & 0.03 \\
\hline Gondoic acid & $20: 1 n-9 c$ & $0.97^{a}$ & $2 \cdot 18$ & $0.51^{a}$ & 0.27 & -1.90 & $0.39^{a}$ & 0.14 & 0.08 \\
\hline$n-6$ PUFA & & $19 \cdot 58^{a}$ & $3 \cdot 30$ & $17 \cdot 68^{a}$ & $2 \cdot 29$ & $-1 \cdot 11$ & $18 \cdot 47^{a}$ & $4 \cdot 80$ & 0.09 \\
\hline Linoleic acid & $18: 2 n-6 c$ & $9.57^{\mathrm{a}}$ & $1 \cdot 31$ & $7 \cdot 90^{\mathrm{b}}$ & 1.07 & $-1 \cdot 21$ & $8.03^{b}$ & 1.58 & 0.01 \\
\hline Eicosadienoic acid & $20: 2 n-6 c$ & $0 \cdot 22^{\mathrm{a}}$ & 0.061 & $0.32^{b}$ & 0.14 & +1.45 & $0 \cdot 28^{a, b}$ & 0.10 & 0.03 \\
\hline DGLA & $20: 3 n-6 c$ & $2 \cdot 03^{a}$ & $1 \cdot 14$ & $2 \cdot 98^{\mathrm{b}}$ & 0.91 & +1.47 & $2 \cdot 67^{a, b}$ & 0.86 & 0.01 \\
\hline $\mathrm{AA}$ & $20: 4 n-6 c$ & $7 \cdot 76^{a}$ & 2.51 & $6 \cdot 49^{\mathrm{a}}$ & $1 \cdot 71$ & $-1 \cdot 20$ & $7 \cdot 49^{\mathrm{a}}$ & $3 \cdot 38$ & 0.21 \\
\hline$n-3$ PUFA & & $10 \cdot 20^{a}$ & 6.03 & $19 \cdot 50^{b}$ & $8 \cdot 41$ & +1.91 & $17 \cdot 12^{b}$ & 9.07 & 0.002 \\
\hline ETE & $20: 3 n-3 c$ & $0 \cdot 19^{a}$ & 0.08 & $0.65^{\mathrm{b}}$ & $1 \cdot 32$ & +3.42 & $0.28^{a}$ & 0.14 & 0.04 \\
\hline EPA & $20: 5 n-3 c$ & $0 \cdot 83^{a}$ & 0.32 & $0.83^{a}$ & 0.55 & & $0.85^{a}$ & 0.35 & 0.94 \\
\hline DPA & $22: 5 n-3 c$ & $6 \cdot 94^{a}$ & $5 \cdot 82$ & $16 \cdot 8^{\mathrm{b}}$ & 8.78 & $+2 \cdot 42$ & $14.50^{\mathrm{b}}$ & $9 \cdot 37$ & 0.0005 \\
\hline $\mathrm{DHA}$ & $22: 6 n-3 c$ & $2 \cdot 24^{a}$ & 1.81 & $1 \cdot 24^{b}$ & 0.71 & -1.81 & $1.49^{a, b}$ & $1 \cdot 18$ & 0.04 \\
\hline Total trans & & $0.39^{a}$ & 0.11 & $0.53^{a}$ & 0.22 & $+1 \cdot 36$ & $0.46^{\mathrm{a}}$ & 0.12 & 0.08 \\
\hline \multirow[t]{2}{*}{ Elaidic acid } & $18: 1 n-9 t$ & $0 \cdot 23^{a}$ & 0.08 & $0.29^{a}$ & 0.14 & $+1 \cdot 26$ & $0 \cdot 26^{a}$ & 0.12 & 0.25 \\
\hline & $20: 4 n-6 t$ & $0 \cdot 16^{a}$ & 0.05 & $0.24^{b}$ & 0.11 & +1.50 & $0 \cdot 20^{a, b}$ & 0.06 & 0.009 \\
\hline Oleic acid:stearic acid ratio & & $1.03^{a}$ & $0 \cdot 10$ & $1.04^{a}$ & 0.12 & & $1 \cdot 00^{\mathrm{a}}$ & 0.09 & 0.93 \\
\hline SFA:MUFA ratio & & $2 \cdot 58^{a}$ & 0.32 & $2 \cdot 45^{\mathrm{a}}$ & 0.31 & -1.05 & $2 \cdot 58^{a}$ & 0.30 & 0.31 \\
\hline AA:DGLA ratio & & $5 \cdot 02^{\mathrm{a}}$ & 2.99 & 2.50 & 1.42 & -2.01 & $3 \cdot 26^{a, b}$ & $2 \cdot 30$ & 0.01 \\
\hline AA:DHA ratio & & $4 \cdot 34^{a}$ & 1.63 & $6 \cdot 09^{b}$ & $2 \cdot 14$ & +1.40 & $6 \cdot 04^{\mathrm{b}}$ & $2 \cdot 35$ & 0.02 \\
\hline Linoleic acid:DPA ratio & & $2 \cdot 24^{a}$ & 1.66 & $0.66^{b}$ & 0.45 & -3.39 & $0.93^{a, b}$ & 0.76 & 0.019 \\
\hline Linoleic acid:ETE ratio & & $56 \cdot 19^{a}$ & $16 \cdot 80$ & $33 \cdot 36^{\mathrm{b}}$ & $20 \cdot 13$ & -1.68 & $39 \cdot 39^{\mathrm{b}}$ & $33 \cdot 13$ & 0.0012 \\
\hline$n-6: n-3$ ratio & & $2 \cdot 41^{\mathrm{a}}$ & $1 \cdot 16$ & $1 \cdot 14^{b}$ & 0.65 & $-2 \cdot 11$ & $1.45^{\mathrm{b}}$ & 0.89 & 0.02 \\
\hline
\end{tabular}

DGLA, dihomo- $\gamma$-linolenic; AA, arachidonic acid; ETE, eicosatrienoic acid; DPA, docosapentaenoic acid.

a,b Mean values within a row with unlike superscript letters were significantly different $(P<0.05$; Dunn's test).

${ }^{*}$ Data are expressed as a percentage of peak areas in the gas chromatograms. The peaks were identified using authentic references, and the identified peaks were $>97 \%$

of the total GC peaks. Evaluated by the Kruskal-Wallis test with Dunn's multiple comparison test.

$\dagger$ Fold increase or decrease $(+/-)$ between controls and CD patients before the GFD intervention. 
transformed into palmitoleic acid $(16: 1)$, the concentrations of which were $1 \cdot 22$-fold higher than those in healthy subjects. However, the oleic acid:stearic acid ratio, related to endogenous synthesis of MUFA, did not change significantly in CD patients when compared with that in healthy subjects.

As regards the PUFA $n-6$ family, the concentrations of linoleic acid were 1.21-fold lower, whereas those of its derivative DGLA were 1.47-fold higher in CD patients than in healthy subjects, indicating increased desaturation and elongation of the $n-6$ FA family. The increase in DGLA concentrations was associated with a $1 \cdot 20$-fold lower concentration of arachidonic acid (AA).

To determine the efficiency of PUFA synthesis from their precursors in terms of desaturase activity, the AA:DGLA ratio was calculated. This ratio was $2 \cdot 01$-fold significantly lower in $\mathrm{CD}$ patients on an unrestricted diet $(P<0 \cdot 01)$, suggesting either an inhibition of $\Delta 5$-desaturase activity or an augmentation of $\Delta 6$-desaturase activity. The putative inhibition of $\Delta 5$-desaturase activity or augmentation of $\Delta 6$-desaturase activity was also observed after 1 year of GFD intervention.

As regards the $n-3$ series, the concentrations of DHA were 1.81-fold lower in $\mathrm{CD}$ patients than in healthy subjects $(P=0.04)$, while those of eicosatrienoic acid and docosapentaenoic acid were significantly higher in CD patients than in healthy subjects (3.42-fold and 2.42-fold, respectively).

The $n-6: n-3$ ratio, expressed as AA:DHA ratio, is considered to be a marker of inflammation. In the present study, a significant 1.40 -fold increase in this ratio was observed in newly diagnosed CD patients, and it appeared to be mainly due to significantly lower concentrations of DHA in CD patients than in healthy subjects, while the concentrations of AA were quite similar. This could underline that $\mathrm{CD}$ patients have an intrinsic FA abnormality.

A compensatory significantly higher total content of the trans-family FA was also observed in CD patients when compared with healthy subjects, in particular, the increase observed in $(20: 4 t) \mathrm{FA}$ content, which was 1.50 -fold higher in $\mathrm{CD}$ patients on an unrestricted diet than in healthy subjects $(P=0.009)$.

CD patients were evaluated for lipidomic profile 1 year after GFD intervention. FA concentrations differed significantly from those of controls. Significantly lower concentrations of palmitic acid, cis-vaccenic acid and linoleic acid and higher concentrations of docosapentaenoic acid were observed. After 1 year of GFD intervention, the AA:DGLA ratio was still lower than that in healthy subjects, thus suggesting a persistent inhibition of desaturase activity up to 1 year of GFD intervention. On comparison of CD patients before and after the GFD intervention, the AA:DHA ratio was found to be unaltered after dietetic treatment, putting in evidence a persistent inflammation state. Overall, no differences were found in FA profile apart from the concentrations of arachidic acid (20:0), which were significantly lower in CD patients on a GFD for 1 year than in those on an unrestricted diet $(P=0 \cdot 02)$. No correlation between any of the FA and the inflammatory parameters was found in the study groups (data not shown).

\section{Discussion}

The main aims of the present study were to perform a lipidomic evaluation of FA content in the erythrocyte membranes of CD patients and to investigate its relationship with inflammation as well as the effects of a dietetic treatment without gluten. The results indicated that a pro-inflammatory FA profile was present in CD patients and that 1-year GFD intervention was not sufficient to restore FA concentrations to normality. Fig. 3 depicts standard de novo and elongation/ desaturation reactions involved in FA biosynthesis and indicates where CD patients differ from healthy subjects.

In agreement with these observations, increased circulating concentrations of IL-6 and IL-8, classical mediators of inflammation, were found in CD patients when compared with healthy subjects. This evidence sustained once more the systemic inflammatory component of the disease ${ }^{(21)}$. These inflammation-related biomarkers also seem to be actively involved in slow inflammatory processes and to promptly respond to GFD interventions, further supporting the notion that the dietary treatment induces anti-inflammatory changes ${ }^{(22)}$.

FA can influence inflammation through a variety of mechanisms, including actions via cell-surface and intracellular receptors/sensors that control inflammatory cell signalling and gene expression patterns ${ }^{(23)}$. Patients with active CD exhibited a significant increase in the concentrations of arachidic acid, a SFA, when compared with controls. In addition, this SFA exhibited a significant decrease in concentrations after 1 year of GFD intervention, acting differently from the other SFA, which were present at lower concentrations in CD patients than in healthy subjects and substantially unaffected by diet. This feature could make arachidic acid a potential biomarker of CD. Another remarkable finding from the present study was the partial transformation of SFA into MUFA. SFA can elicit desaturase activity. In particular, the transformation of palmitic acid into palmitoleic acid was prominent in CD patients with a reduction of palmitic acid concentrations and an elevation of palmitoleic acid concentrations in comparison with healthy subjects. This elevation was not evident when the total MUFA were considered, the concentrations of which were lower than those in healthy subjects. The oleic acid:stearic acid ratio, representative of the MUFA endogenous synthesis, also did not exhibit significant differences among the study groups. Discrepancies between the behaviours of single FA and their class were also a distinctive trait for other groups of FA, such as $n-6$ and $n-3$ FA. This evidence may reflect a different role and involvement of each single FA in the evolution of CD.

As regards n-6 PUFA, overall this class of FA existed at lower concentrations in $\mathrm{CD}$ patients than in healthy subjects. The essential FA linoleic acid was found at lower concentrations in patients with active disease than in healthy subjects. Data reported in the literature have already demonstrated that the concentrations of this FA were significantly lower in the serum of patients with 'chronic intestinal failure' as well as $\mathrm{CD}^{(4,14,24)}$. Steel et al. ${ }^{(14)}$ reported low concentrations of linoleic acid in the intestinal mucosa of CD patients, both before and after the GFD intervention. Our data are consistent with 

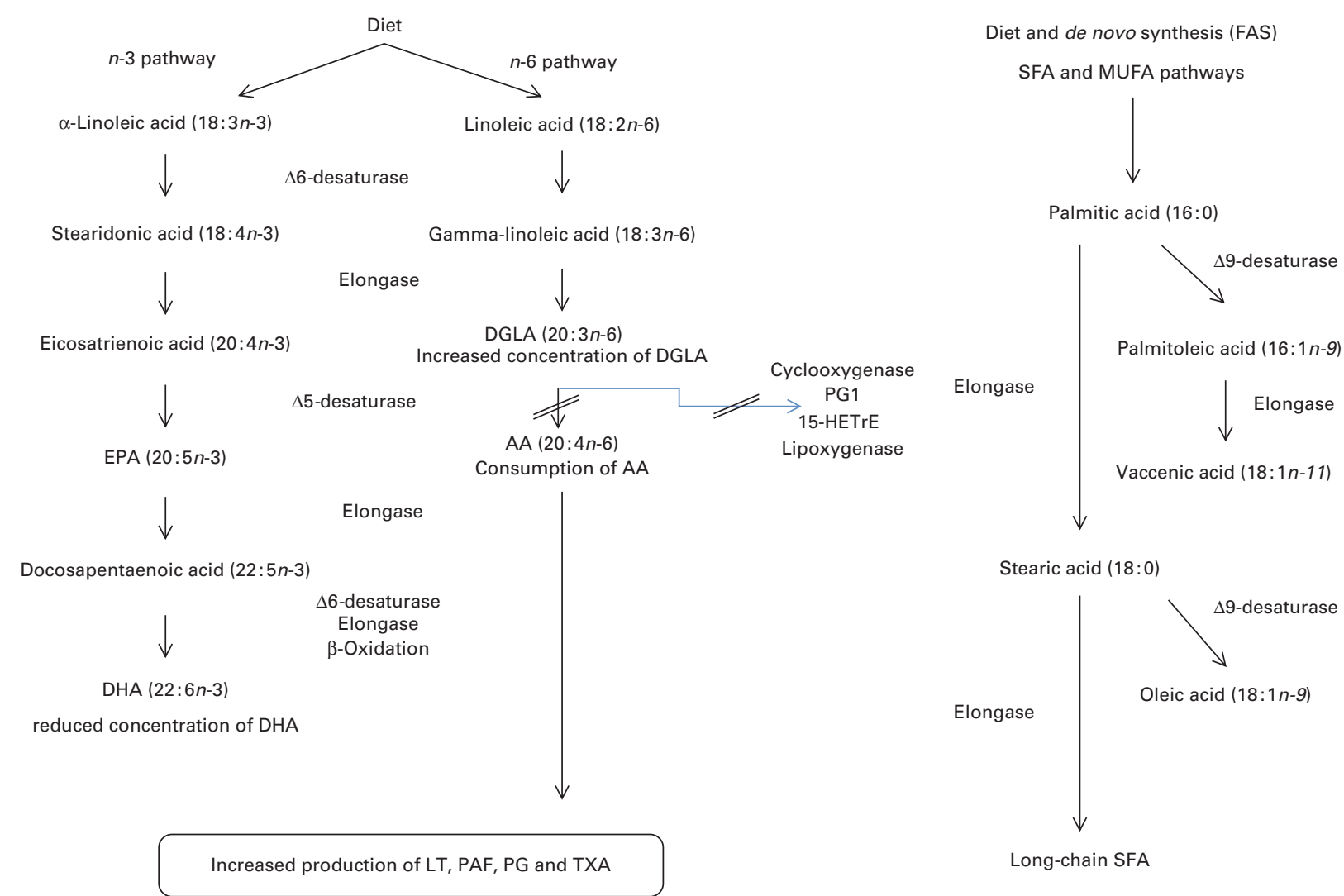

Fig. 3. The standard de novo and elongation/desaturation reactions involved in fatty acid biosynthesis. The figure puts in evidence where coeliac disease (CD) patients differ from healthy subjects. The reduction of arachidonic acid (AA) concentrations in CD patients, restored by 1-year gluten-free diet intervention, suggests a different utilisation of dihomo- $\gamma$-linolenic acid (DGLA) as a substrate for other enzymes and a decrease in the conversion of DGLA into AA. FAS, fatty acid synthase; HETrE, hydroxyeicosatetraenoic acid; LT, leukotrienes; PAF, platelet-activating factors; TXA, thromboxane. (A colour version of this figure can be found online at http://www.journals.cambridge.org/bjn)

this report as the linoleic acid content in the erythrocyte membranes of CD patients after 1 year of GFD intervention was still lower when compared with that of healthy subjects.

CD patients exhibited significantly higher DGLA concentrations, along with moderate and not significantly lower AA concentrations, in comparison with healthy subjects. DGLA derives from linoleic acid and is converted into AA. Both of them are substrates for the peroxidase enzymes (cyclooxygenase) that transform these FA into PGE1 and PGE2, respectively ${ }^{(25)}$. Another metabolic DGLA pathway involves the peroxidation enzymes (lipoxygenase) naturally existing in neutrophils, macrophages, monocytes and epithelial cells, which can inhibit the synthesis of pro-inflammatory metabolites from AA. The inhibition of these metabolites (leukotrienes) is considered to be the main mechanism for the anti-inflammatory action of DGLA. A possible block in DGLA metabolism, including the pathway leading to the conversion into AA, could activate the synthesis of these pro-inflammatory metabolites (such as PGE2, leukotrienes and platelet-activating factors) from $\mathrm{AA}^{(22)}$.

An alternative pathway could also be hypothesised, considering that DGLA has a series of important functions as a precursor for eicosanoids (e.g. PGE1) or other mediators showing a regulatory activity in immune-related cell reactions. In particular, the reduction of $\mathrm{AA}$ concentrations in $\mathrm{CD}$ patients, restored by 1-year GFD intervention, suggests a different utilisation of DGLA as a substrate for other enzymes and a decrease in the conversion of DGLA into AA (via $\Delta 5$-desaturase). The significant elevation of trans-FA content in $\mathrm{CD}$ patients in comparison with healthy subjects could also explain the present findings. The elevation of the content of trans-FA is harmful as these FA heavily interfere with the desaturation of both $n-6$ and $n-3$ FA, reducing, in this way, the conversion of DGLA into AA and the availability of EPA and $\mathrm{DHA}^{(26,27)}$.

Solakivi et al. ${ }^{(4)}$ evaluated serum FA profile in CD patients before and after 1 year of GFD intervention and found a decrease in the concentrations of AA and the $n-3$ PUFA EPA and DHA and a slight increase after GFD intervention in patients relative to controls. In the present study, significantly higher total concentrations of $n$ - 3 PUFA were found in CD patients than in healthy subjects. However, this was not true for DHA, which was present at lower concentrations in CD patients than in healthy subjects.

DHA is a precursor of resolvins; these substances are antiinflammatory mediators that could be pivotal in the evolution of CD. Besides, the low concentrations of DHA in CD patients, not completely restored by 1-year GFD intervention, could be due to a reduced conversion of docosapentaenoic acid into DHA. Probably, this is a consequence of the alterations 
in elongase and $\Delta 6$-desaturase activities ${ }^{(28)}$. Therapeutically, this evidence may show interesting implications in the management of CD. Supplementation with DHA is gaining increasing importance for the treatment of different pathological conditions, as it seems to be effective at countering the pro-inflammatory effects of AA metabolites (e.g. cyclooxygenase 2 expression or the release of PGE2 and IL-8) ${ }^{(29)}$.

It is well known that a high $n-6: n-3$ ratio predisposes to inflammation ${ }^{(30-32)}$. In this connection, when the AA:DHA ratio was calculated, its value was found to be significantly higher in $\mathrm{CD}$ patients, either before or after the GFD intervention, than in healthy subjects. This evidence lets us hypothesise, in agreement with data reported in the literature $^{(14)}$, that intrinsic abnormalities in FA profile could contribute to inflammation in CD patients.

Furthermore, trans-FA concentrations were higher in CD patients than in healthy subjects. In particular, the concentrations of trans-FA (20:4) isomers were markedly elevated in $\mathrm{CD}$ patients. It is known that these isomers can be formed only during the free radical stress conditions, which are likely to occur during inflammatory disorders ${ }^{(11,33,34)}$. Although preliminary, these data on trans-FA isomers, indicative of the formation of sulphur-centred radical species, suggest that these parameters may serve to follow up radical stress involving lipids in $C D$ patients ${ }^{(35)}$. This information can be useful for further studies, especially coupling proteomic and lipidomic studies, which could give more information of tandem damages influencing the disease outcome.

Finally, the effects of GFD on FA profile were evaluated after 1 year of dietetic treatment. Notably, the different classes of FA reacted differently to the GFD intervention, showing that the treatment was not able to restore FA concentrations to those observed in healthy subjects. Taken as a whole, the present results let us hypothesise that 1-year GFD intervention may be not sufficient to achieve a significant restoration in intestinal functions and absorption, and hence dietetic treatment for a prolonged period might be needed to achieve a significant improvement of FA profiles.

\section{Conclusions}

The lipidomic analysis of erythrocyte membranes confirmed the presence of an altered FA composition in CD patients. Moreover, an appropriate dietary treatment with exclusion of gluten can modify FA profile, even if 1-year GFD intervention seems to be not sufficient to restore FA concentrations to normality, suggesting an inadequate supply of long-chain PUFA $^{(4)}$. Lastly, this procedure, being easier and non-invasive compared with the evaluation of the FA pattern of the intestinal mucosa, could offer more potentiality for also evaluating therapeutic interventions in $\mathrm{CD}$ patients by using FA supplementation.

\section{Acknowledgements}

The authors thank Dr Antonella Giannini (IRCCS 'Saverio de Bellis') for her valuable technical assistance and Dr Valentina
Sunda and Mr Simone Deplano (Lipinutragen, Bologna) for their valuable assistance in FA analysis.

The present study was supported by the Italian Ministry of Health (grant no. 254-16.05.2012).

The authors' contributions are as follows: G. R., C. F. and F. R. were responsible for data analysis and interpretation, wrote the manuscript and contributed to the intellectual content of the article; A. O., M. M. and B. D. collected and interpreted the data and made intellectual contributions to the article. All authors participated in the study to a significant extent and read and approved the submitted manuscript.

C. F. discloses her position as a partner of Lipinutragen srl. The other authors declare no competing interests.

\section{References}

1. Gujral N, Freeman HJ \& Thomson ABR (2012) Celiac disease: prevalence, diagnosis, pathogenesis and treatment. World J Gastroenterol 18, 6036-6059.

2. Haines ML, Anderson RP \& Gibson PR (2008) Systematic review: the evidence base for long-term management of coeliac disease. Aliment Pharmacol Ther 28, 1042-1066.

3. Zhao Q (2009) Inflammation, autoimmunity, and atherosclerosis. Discov Med 8, 7-12.

4. Solakivi T, Kaukinen K, Kunnas T, et al. (2009) Serum fatty acid profile in celiac disease patients before and after a gluten-free diet. Scand J Gastroenterol 44, 826-830.

5. West J, Logan RFA, Card TR, et al. (2004) Risk of vascular disease in adults with diagnosed coeliac disease: a population-based study. Aliment Pharmacol Ther 20, 73-79.

6. Pitocco D, Giubilato S, Martini F, et al. (2011) Combined atherogenic effects of celiac disease and type 1 diabetes mellitus. Atherosclerosis 217, 531-535.

7. Hunter JE, Zhang J \& Kris-Etherton PM (2010) Cardiovascular disease risk of dietary stearic acid compared with trans, other saturated, and unsaturated fatty acids: a systematic review. Am J Clin Nutr 91, 46-63.

8. Chatgilialoglu C, Ferreri C, Lykakis IN, et al. (2006) Transfatty acids and radical stress: what are the real culprits? Bioorg Med Chem 14, 6144-6148.

9. Ferretti G, Bacchetti T, Masciangelo S, et al. (2012) Celiac disease, inflammation and oxidative damage: a nutrigenetic approach. Nutrients 4, 243-257.

10. Wolf C \& Quinn PJ (2008) Lipidomics: practical aspects and applications. Prog Lipid Res 47, 15-36.

11. Ferreri C \& Chatgilialoglu C (2012) Role of fatty acid-based functional lipidomics in the development of molecular diagnostic tools. Expert Rev Mol Diagn 12, 767-780.

12. Kim YJ, Kim E-H \& Hahm KB (2012) Oxidative stress in inflammation-based gastrointestinal tract diseases: challenges and opportunities. J Gastroenterol Hepatol 27, 1004-1010.

13. Pala V, Krogh V, Muti P, et al. (2001) Erythrocyte membrane fatty acids and subsequent breast cancer: a prospective Italian study. J Natl Cancer Inst 93, 1088-1095.

14. Steel DM, Ryd W, Ascher H, et al. (2006) Abnormal fatty acid pattern in intestinal mucosa of children with celiac disease is not reflected in serum phospholipids. J Pediatr Gastroenterol Nutr 43, 318-323.

15. Antonioli DA (2003) Celiac disease: a progress report. Mod Pathol 16, 342-346.

16. Tremblay AJ, Morrissette H, Gagne J-M, et al. (2004) Validation of the Friedewald formula for the determination of low-density 
lipoprotein cholesterol compared with beta-quantification in a large population. Clin Biochem 37, 785-790.

17. Bligh EG \& Dyer WJ (1959) A rapid method of total lipid extraction and purification. Can J Biochem Physiol 37, 911-917.

18. Aoun M, Feillet-Coudray C, Fouret G, et al. (2012) Rat liver mitochondrial membrane characteristics and mitochondrial functions are more profoundly altered by dietary lipid quantity than by dietary lipid quality: effect of different nutritional lipid patterns. Br J Nutr 107, 647-659.

19. Kramer JK, Fellner V, Dugan ME, et al. (1997) Evaluating acid and base catalysts in the methylation of milk and rumen fatty acids with special emphasis on conjugated dienes and total trans fatty acids. Lipids 32, 1219-1228.

20. Brenner RR (1989) Factors influencing fatty acid chain elongation and desaturation. In The Role of Fats in Human Nutrition, 2nd ed., pp. 46-79 [AJ Vergroesen and M Crawford, editors]. London: Academic Press.

21. Fornari MC, Pedreira S, Niveloni S, et al. (1998) Pre- and post-treatment serum levels of cytokines IL-1beta, IL-6, and IL-1 receptor antagonist in celiac disease. Are they related to the associated osteopenia? Am J Gastroenterol 93 , 413-418.

22. Russo F, Chimienti G, Clemente C, et al. (2013) Adipokine profile in celiac patients: differences in comparison with patients suffering from diarrhea-predominant IBS and healthy subjects. Scand J Gastroenterol 48, 1377-1385.

23. Calder PC (2011) Fatty acids and inflammation: the cutting edge between food and pharma. Eur J Pharmacol 668 Suppl. 1, S50-S58.

24. Chambrier C, Garcia I, Bannier E, et al. (2002) Specific changes in $n-6$ fatty acid metabolism in patients with chronic intestinal failure. Clin Nutr 21, 67-72.

25. Wang X, Lin H \& Gu Y (2012) Multiple roles of dihomo$\gamma$-linolenic acid against proliferation diseases. Lipids Health Dis 11, 25.
26. Simopoulos AP (1997) Omega-6/omega-3 fatty acid ratio and trans fatty acids in non-insulin-dependent diabetes mellitus. Ann N Y Acad Sci 827, 327-338.

27. Simopoulos AP (2009) Omega-6/omega-3 essential fatty acids: biological effects. World Rev Nutr Diet 99, 1-16.

28. Kaur G, Cameron-Smith D, Garg M, et al. (2011) Docosapentaenoic acid $(22: 5 n-3)$ : a review of its biological effects. Prog Lipid Res 50, 28-34.

29. Vincentini O, Quaranta MG, Viora M, et al. (2011) Docosahexaenoic acid modulates in vitro the inflammation of celiac disease in intestinal epithelial cells via the inhibition of CPLA2. Clin Nutr 30, 541-546.

30. Riediger ND, Othman R, Fitz E, et al. (2008) Low n-6:n-3 fatty acid ratio, with fish- or flaxseed oil, in a high fat diet improves plasma lipids and beneficially alters tissue fatty acid composition in mice. Eur J Nutr 47, 153-160.

31. Pischon T, Hankinson SE, Hotamisligil GS, et al. (2003) Habitual dietary intake of $n-3$ and $n-6$ fatty acids in relation to inflammatory markers among US men and women. Circulation 108, 155-160.

32. Tulk HM \& Robinson LE (2009) Modifying the $n-6 / n-3$ polyunsaturated fatty acid ratio of a high-saturated fat challenge does not acutely attenuate postprandial changes in inflammatory markers in men with metabolic syndrome. Metabolism 58, 1709-1716.

33. Ferreri C, Angelini F, Chatgilialoglu C, et al. (2005) Trans fatty acids and atopic eczema/dermatitis syndrome: the relationship with a free radical cis-trans isomerization of membrane lipids. Lipids 40, 661-667.

34. Chatgilialoglu C, Ferreri C, Melchiorre M, et al. (2014) Lipid geometrical isomerism: from chemistry to biology and diagnostics. Chem Rev 114, 255-284.

35. Chatgilialoglu C, Ferreri C, Torreggiani A, et al. (2011) Radiation-induced reductive modifications of sulfurcontaining amino acids within peptides and proteins. J Proteomics 74, 2264-2273. 